\title{
Converting an odds ratio to a range of plausible relative risks for better communication of research findings
}

In this Research Methods and Reporting article (BMJ 2014;348:f7450, doi:10.1136/bmj.f7450), the box "Definitions of odds and risks" contains some errors in how the alphabetical symbols of a, b, c, and d have been applied. Firstly, in the list of bullet points under "Definitions," the "Total for control" category should have been assigned the letters b+d, not "c+d" as was published.

Also, under "An applied example" the letters b and c that were assigned to the second and third bullet point are the wrong way round. The second bullet point, "Number of participants who did not quit at four weeks after the hospital based intervention: 162 ," should have been marked c, not "b." While the third bullet point, "Number of participants who quit at four weeks after usual care: 37," should have been assigned b, not "c."

Finally, the last bullet point in that list under "An applied example," which reads "Total for usual care: 224 participants," should have said $b+d$, not "c+d" as was published.

Cite this as: BMJ 2014;348:g2124

๑ BMJ Publishing Group Ltd 2014 